\title{
molecules
}

ISSN 1420-3049

www.mdpi.com/journal/molecules

Article

\section{Interference of Phenylethanoid Glycosides from Cistanche tubulosa with the MTT Assay}

\author{
Yu-Jie Wang ${ }^{1, *}$, Si-Min Zhou ${ }^{2}$, Gang $\mathrm{Xu}^{2}$ and Yu-Qi Gao ${ }^{2}$ \\ 1 College of Ethnomedicine, Chengdu University of Traditional Chinese Medicine, \\ Chengdu 611137, China \\ 2 College of High Altitude Military Medicine, Third Military Medical University, \\ Chongqing 400038, China; E-Mails: lifengguang119@163.com (S.-M.Z.); \\ xugangyjy@126.com (G.X.); gaoyuqiyjy@126.com (Y.-Q.G.) \\ * Author to whom correspondence should be addressed; E-Mail: superwangyj@126.com; \\ Tel./Fax: +86-28-6180-0094.
}

Academic Editor: Derek J. McPhee

Received: 2 February 2015 / Accepted: 28 April 2015 / Published: 5 May 2015

\begin{abstract}
The MTT assay, as a screening method, has been widely used to measure the viability and proliferation of cells. However, it should be noted that MTT assay may not accurately reflect the effect of Cistanche tubulosa ethanolic extract on EA.hy926 cells viability. To investigate and identity the components responsible for the contradictory observations of the MTT assay, echinacoside and acteoside, two main phenylethanoid glycosides, from $C$. tubulosa ethanolic extract were isolated. The data derived from CCK-8, Hoechst 33342 and annexin V-FITC/PI assays suggest that the caffeyl group present in both isolated compounds was responsible for the conflicting results of the MTT assay. These data emphasize the need of using a variety of different methods to determine the effect of medicinal agents on cell viability to avoid generating misleading results.
\end{abstract}

Keywords: MTT assay; phenylethanoid glycoside; caffeic acid; cell viability; interference

\section{Introduction}

The parasitic plant Cistanche tubulosa (Schrenk) R. Wight (Orobanchaceae family) is widely distributed in North African, Arab, and Asian countries [1]. The stems from C. tubulosa and C. deserticola 
are important in traditional Chinese medicine, having been used for the treatment of impotence, sterility, lumbago, and constipation due to colonic inertia [2]. In addition, the ethanolic extract of C. tubulosa has been shown to have a significant protective effect against cerebral hypoxia in mice [3].

Endothelial cells play a crucial role in the pathogenesis of hypoxic pulmonary hypertension. The EA.hy926 endothelial cell line is considerably similar to primary endothelial cells. During our own study of the anti-hypoxic effect of C. tubulosa ethanolic extract on EA.hy926 endothelial cell, we noticed that the 3-(4,5-dimethylthiazol-2-yl)-2,5-diphenyltetrazolium bromide (MTT) assay, which is commonly used to assess cell viability in studies of cytotoxicity and cytostatic activity, generated conflicting results by showing increased EA.hy926 cell viability after treatment.

Accurate assessment of cell viability is essential to identify potential cytotoxic or cytoprotective effects of a medicinal agent. We therefore investigated which components present in C. tubulosa ethanolic extract may be responsible for the conflicting results observed with the MTT assay. We isolated two main phenylethanoid glycosides (PhGs) from C. tubulosa ethanolic extract, echinacoside (hereafter compound 1) and acteoside (hereafter compound 2), and determined their effect on EA.hy926 cell viability using a variety of methods. In addition, in order to further clarify which substituent of compound 1 and $\mathbf{2}$ may be responsible for the contradictory results, we tested their aglycones, caffeic acid (hereafter compound 3) and 3,4-dihydroxylphenylethanol (hereafter compound 4), on EA.hy926 cell viability using the same methods.

\section{Results and Discussion}

\subsection{Identification of Compounds}

The structures of the two compounds isolated from C. tubulosa ethanolic extract were identified by nuclear magnetic resonance (NMR) and mass spectrometry (MS) analyses and are shown in Figure 1. Compound 1 was identified as echinacoside by comparison with previously reported NMR (Table 1) and MS $\left(\mathrm{m} / z 785[\mathrm{M}-\mathrm{H}]^{-}\right)$data [4]. Compound 2 NMR spectra were highly similar to those of compound 1, except for the absence of a $\beta$-D-glucopyranosyl group (Table 1). Compound $2\left(\mathrm{~m} / z 623[\mathrm{M}-\mathrm{H}]^{-}\right)$was identified as acteoside [5]. Importantly, both compounds have the same aglycone, which includes caffeic acid (compound 3) and 3,4-dihydroxylphenylethanol (compound 4).

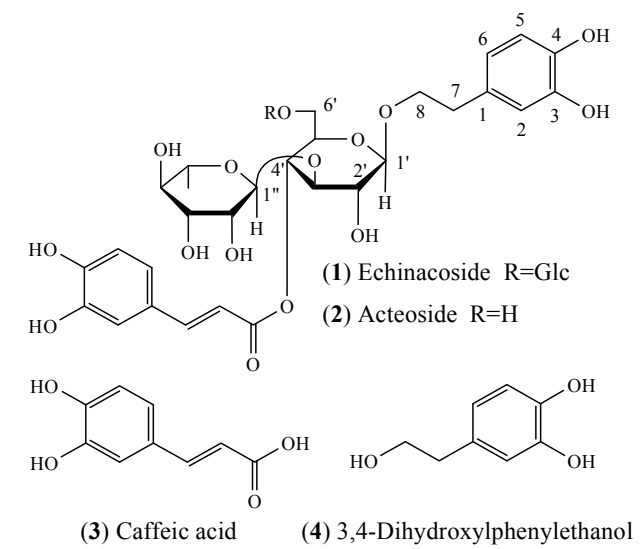

Figure 1. Structures of compounds 1-4. 
Table 1. ${ }^{1} \mathrm{H}(600 \mathrm{MHz})$ and ${ }^{13} \mathrm{C}(150 \mathrm{MHz})$ NMR data of echinacoside (1) and acteoside (2) $\left(\mathrm{CD}_{3} \mathrm{OD}, \delta\right.$ in $\left.\mathrm{ppm}\right)$.

\begin{tabular}{|c|c|c|c|c|}
\hline \multirow{2}{*}{ Position } & \multicolumn{2}{|c|}{ Echinacoside } & \multicolumn{2}{|c|}{ Acteoside } \\
\hline & $\delta_{\mathrm{H}}(J$ in $\mathrm{Hz})$ & $\boldsymbol{\delta}_{\mathrm{C}}$ & $\delta_{\mathrm{H}}(J$ in Hz $)$ & $\delta_{\mathrm{C}}$ \\
\hline 1 & & 130.1 & & 130.1 \\
\hline 2 & $6.69(\mathrm{~d}, 2.0)$ & 115.8 & $6.69(\mathrm{~d}, 2.0)$ & 115.8 \\
\hline 3 & & 144.7 & & 144.7 \\
\hline 4 & & 143.3 & & 143.3 \\
\hline 5 & $6.67(\mathrm{~d}, 8.0)$ & 115.0 & $6.67(\mathrm{~d}, 8.0)$ & 115.0 \\
\hline 6 & $6.57(\mathrm{dd}, 1.9,8.0)$ & 119.9 & $6.56(\mathrm{dd}, 1.9,8.0)$ & 119.9 \\
\hline 7 & $2.79(\mathrm{~m}, 2 \mathrm{H})$ & 35.2 & $2.79(\mathrm{~m}, 2 \mathrm{H})$ & 35.2 \\
\hline \multirow[t]{2}{*}{8} & $3.75(\mathrm{~m})$ & 70.1 & $3.73(\mathrm{~m})$ & 70.7 \\
\hline & $4.03(\mathrm{~m})$ & & $4.04(\mathrm{~m})$ & \\
\hline & & 8-O-Glc & & \\
\hline $1^{\prime}$ & $4.38(\mathrm{~d}, 7.9)$ & 102.8 & $4.37(\mathrm{~d}, 7.9)$ & 102.8 \\
\hline $2^{\prime}$ & $3.39(\mathrm{dd}, 8.2,9.2)$ & 74.8 & $3.39(\mathrm{dd} 7.9,9.2)$ & 74.8 \\
\hline $3^{\prime}$ & $3.81(\mathrm{t}, 9.2)$ & 80.3 & $3.81(\mathrm{t}, 9.2)$ & 80.3 \\
\hline $4^{\prime}$ & $5.00(\mathrm{dd}, 9.7,9.7)$ & 69.2 & $4.91(t, 9.7,9.8)$ & 69.2 \\
\hline $5^{\prime}$ & $3.71(\mathrm{~m})$ & 73.4 & $3.59(\mathrm{~m})$ & 74.6 \\
\hline \multirow[t]{2}{*}{$6^{\prime}$} & $3.64(\mathrm{~m})$ & 68.0 & $3.52(\mathrm{~m})$ & 60.9 \\
\hline & $3.93(\mathrm{~m})$ & & $3.61(\mathrm{~m})$ & \\
\hline \multicolumn{5}{|c|}{ 3'-O-Rha } \\
\hline $1 "$ & $5.18(\mathrm{~d}, 1.8)$ & 101.7 & $5.19(\mathrm{~d}, 1.8)$ & 101.6 \\
\hline $2^{\prime \prime}$ & $3.91(\mathrm{dd}, 1.8,3.0)$ & 71.0 & $3.92(\mathrm{dd}, 1.8,3.0)$ & 71.0 \\
\hline $3 "$ & $3.55(\mathrm{~m})$ & 70.7 & $3.57(\mathrm{~m})$ & 70.8 \\
\hline $4 "$ & $3.28(\mathrm{~m})$ & 72.4 & $3.30(\mathrm{~m})$ & 72.4 \\
\hline $5^{\prime \prime}$ & $3.53(\mathrm{~m})$ & 69.1 & $3.55(\mathrm{~m})$ & 69.1 \\
\hline $6 "$ & $1.08(3 \mathrm{H}, \mathrm{d}, 6.2)$ & 17.1 & $1.09(3 \mathrm{H}, \mathrm{d}, 6.2)$ & 17.1 \\
\hline \multicolumn{5}{|c|}{ 6'-O-Glc } \\
\hline $1 " '$ & $4.29(\mathrm{~d}, 7.7)$ & 103.3 & - & - \\
\hline $2^{\prime \prime \prime}$ & $3.19(\mathrm{~m})$ & 73.7 & - & - \\
\hline 3"' & $3.54(\mathrm{~m})$ & 76.4 & - & - \\
\hline 4"' & $3.26(\mathrm{~m})$ & 70.8 & - & - \\
\hline $5^{\prime \prime \prime}$ & $3.23(\mathrm{~m})$ & 76.5 & - & - \\
\hline 6"' & $3.62(\mathrm{~m})$ & 61.2 & - & - \\
\hline & $3.82(\mathrm{~m})$ & & & \\
\hline \multicolumn{5}{|c|}{ 4'-O-Caf } \\
\hline 1 & & 126.3 & & 126.3 \\
\hline 2 & $7.06(\mathrm{~d}, 2.0)$ & 113.9 & $7.05(\mathrm{~d}, 1.9)$ & 113.9 \\
\hline 3 & & 145.4 & & 145.4 \\
\hline 4 & & 148.4 & & 148.4 \\
\hline 5 & $6.78(\mathrm{~d}, 8.2)$ & 115.2 & $6.78(\mathrm{~d}, 8.0)$ & 115.2 \\
\hline 6 & $6.96(\mathrm{dd}, 1.9,8.2)$ & 121.9 & $6.94(\mathrm{dd}, 1.9,8.0)$ & 121.8 \\
\hline 7 & $7.60(\mathrm{~d}, 15.9)$ & 146.8 & $7.59(\mathrm{~d}, 15.9)$ & 146.6 \\
\hline 8 & $6.27(\mathrm{~d}, 15.9)$ & 113.3 & $6.26(\mathrm{~d}, 15.9)$ & 113.4 \\
\hline 9 & & 167.1 & & 166.9 \\
\hline
\end{tabular}




\subsection{Cell Viability Assays}

MTT and CCK-8 assays were used to analyze the effect of compounds 1-4 on EA.hy926 cell viability. The MTT assay showed that the viability of EA.hy926 cells increased significantly after treatment with 25 and $50 \mu \mathrm{M}$ compound 1, 2, or $\mathbf{3}(166.3 \%$ and $174.1 \%$ for compound $\mathbf{1}, 205.8 \%$ and $224.3 \%$ for compound 2 , and $164.8 \%$ and $189.6 \%$ for compound 3 , respectively; $p<0.05$ ), when compared to control cells (Figure 2A). Furthermore, morphological inspection of the cells determined that treatment with $50 \mu \mathrm{M}$ compound 1, 2, or 3 increased the formation of purple formazan crystals, which are considered to be a direct indication of the proportion of living cells (Figure 3). Conversely, the CCK- 8 assay suggested that cell viability decreased significantly after treatment with $12.5,25$, and $50 \mu \mathrm{M}$ compound $\mathbf{1}, \mathbf{2}$, or $3(75.5 \%, 45.1 \%$, and $43.7 \%$ for compound $\mathbf{1}, 85.5 \%, 51.8 \%$, and $34.3 \%$ for compound 2 , and $64.5 \%, 48.2 \%$, and $36.4 \%$ for compound 3 , respectively; $p<0.05$ ), compared to control cells (Figure 2B). Compound 4 did not affect cell viability in either the MTT or CCK-8 assay. The discrepancy between the results obtained with the two assays suggests that one of the two assays may not accurately reflect the effects of compounds $\mathbf{1}$ and $\mathbf{2}$ on EA.hy926 cell viability.

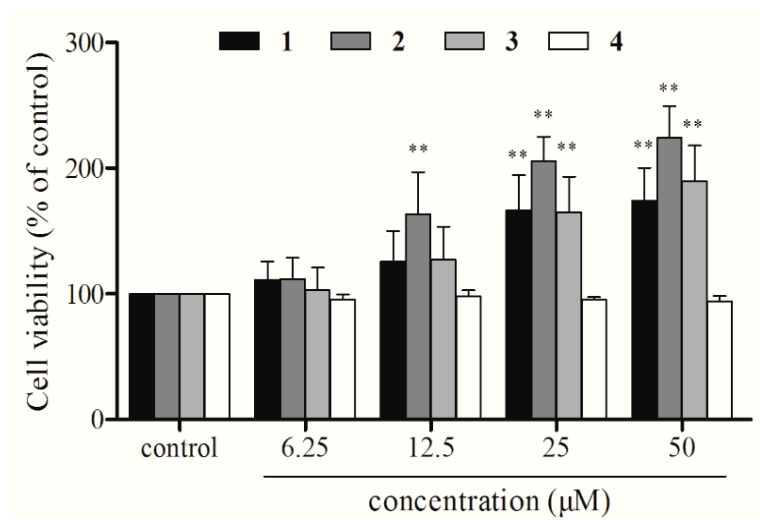

(A)

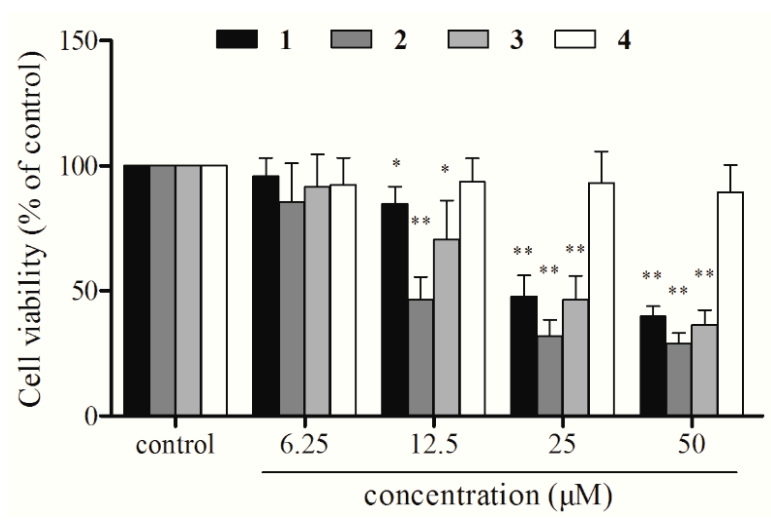

(B)

Figure 2. Effects of compounds 1-4 on cell viability. EA.hy926 cells were treated with 6.25, 12.5, 25 and $50 \mu \mathrm{M}$ echinacoside (1), acteoside (2), caffeic acid (3), 3,4-dihydroxylphenylethanol (4) for $24 \mathrm{~h}$. (A) MTT assay; (B) CCK-8 assay. Data are the mean \pm S.D. of three independent experiments. ${ }^{*} p<0.05$ and $* * p<0.01$ versus control.

\subsection{Apoptosis Assessment by Hoechst Staining}

Hoechst 33342 is a cell-permeable DNA stain that is excited by ultraviolet light and emits blue fluorescence at 460 to $490 \mathrm{~nm}$. The condensed chromatin of apoptotic cells stain more brightly than the chromatin of normal cells [6]. Whereas control cells exhibited uniformly dispersed chromatin, normal organelles, and intact cell membranes, cells incubated with $50 \mu \mathrm{M}$ compounds $\mathbf{1}$, 2, or $\mathbf{3}$ for $48 \mathrm{~h}$ showed the typical staining of apoptotic cells (Figure 4A). Conversely, exposure of cells to $50 \mu \mathrm{M}$ of compound $\mathbf{4}$ did not increase the number of apoptotic nuclei compared to control treatment. 


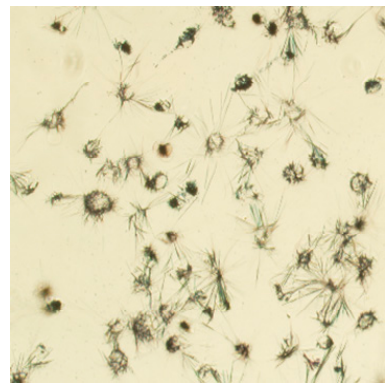

control

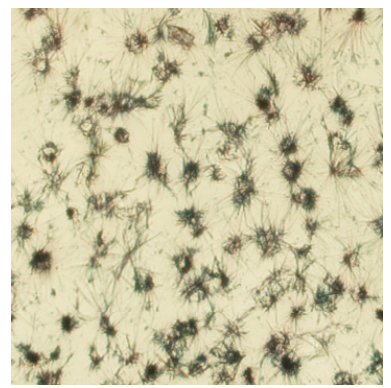

3

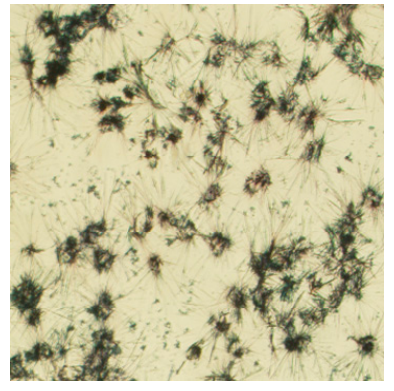

1

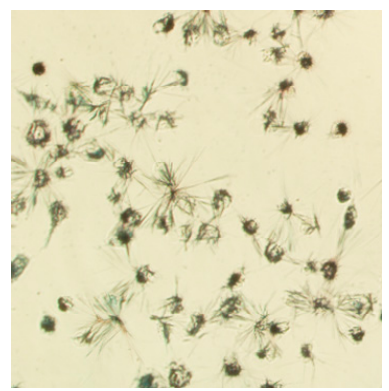

4

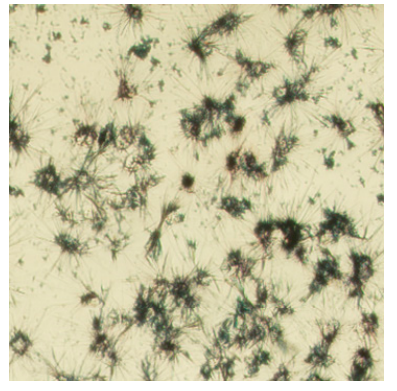

2

Figure 3. Micrographs showing formation of MTT formazan. EA.hy926 cells were treated with $50 \mu \mathrm{M}$ echinacoside (1), acteoside (2), caffeic acid (3), 3,4-dihydroxylphenylethanol (4) for $24 \mathrm{~h}$. Magnification $200 \times$.

A

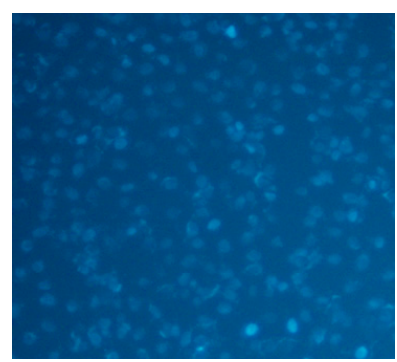

control

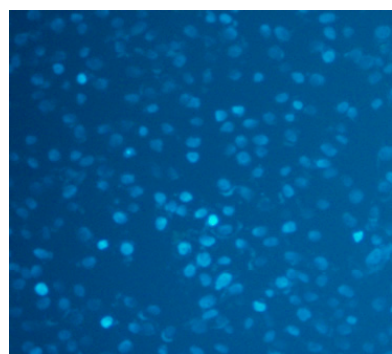

3

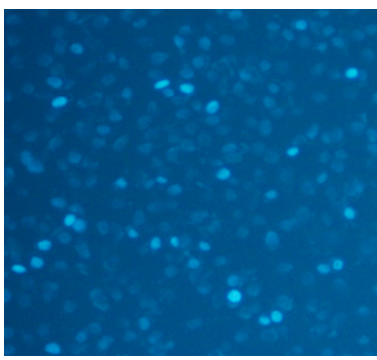

1

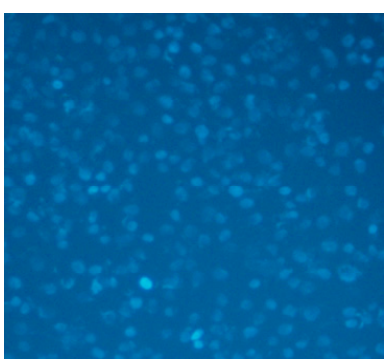

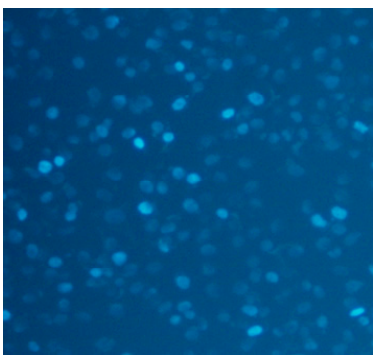

2

Figure 4. Cont. 
B
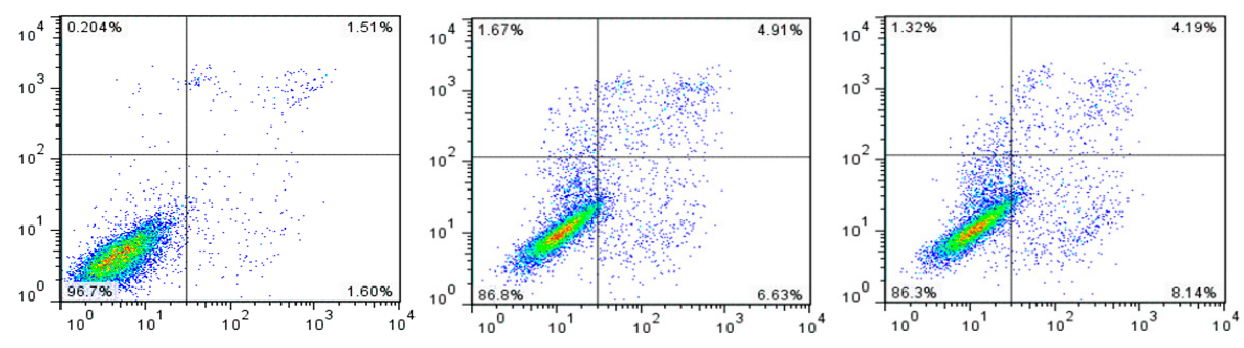

control

1
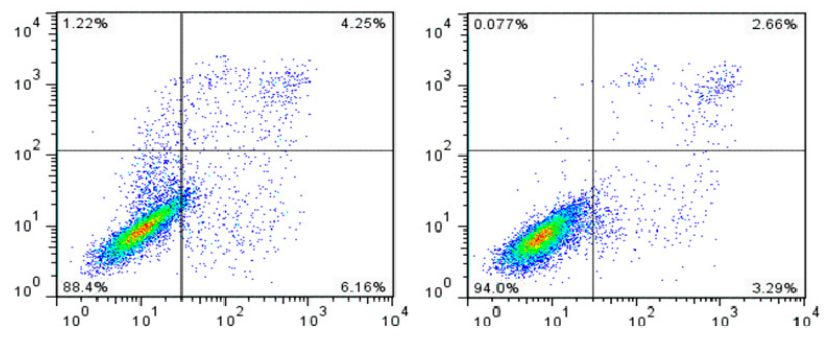

2

3

4

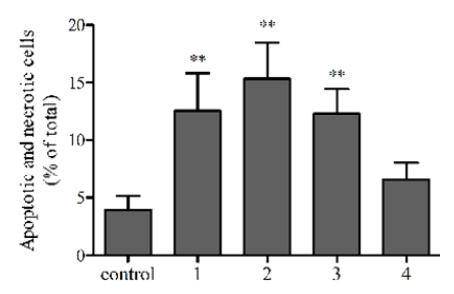

Figure 4. Apoptosis Assessment by Hoechst 33342 Staining and Flow Cytometry. EA.hy926 cells were treated in the absence (control), or in the presence of $50 \mu \mathrm{M}$ echinacoside (1), acteoside (2), caffeic acid (3), 3,4-dihydroxylphenylethanol (4) for $48 \mathrm{~h}$. (A) Apoptotic cells were identified via morphological means after staining of nuclear chromatin with Hoechst 33342 dye. Magnification 200×. (B) Percentage of apoptotic cells in total EA.hy926 cells. Data are the mean \pm S.D. of three independent experiments. ** $p<0.01$ versus control.

\subsection{Flow Cytometric Analysis of Apoptotic Cells}

The annexin V-FITC/PI double-staining assay identifies apoptotic cells by the high affinity binding of annexin V-FITC to phosphatidylserine, which is externalized in cells undergoing apoptosis [7]. In this assay, viable cells do not bind to either annexin V nor PI and are therefore not stained (lower left quadrant), early apoptotic cells are stained green by the binding of annexin V-FITC (lower right quadrant), and late apoptotic cells are stained green and red by the binding of annexin V-FITC to phosphatidylserine and PI to necrotic cells, respectively (upper right quadrant). As shown in Figure 4B, cells incubated with $50 \mu \mathrm{M}$ compounds 1, 2, or 3 for $48 \mathrm{~h}$, the percentage of apoptotic cells increased from $3.74 \%$ (control cells) to $10.32 \%, 12.95 \%$, and $11.30 \%$, respectively. Compared to control cells, compound 4 did not increase the percentage of apoptotic EA.hy926 cells.

Consistent with the results obtained with the CCK-8 assay and by Hoechst 33342 staining, the flow cytometry assay showed that compounds 1, 2, and $\mathbf{3}$ induced apoptosis in EA.hy926 cells. All together, these observations suggest that the increase in EA.hy926 cell viability observed with the MTT assay after treatment with compounds $\mathbf{1}-\mathbf{3}$ represented a false-positive result.

\subsection{Discussion}

In 1983, Mosmann developed a colorimetric MTT microplate assay for measuring cell proliferation and cytotoxicity [8]. This simple assay, and modifications of it, is now extensively used in cell biology 
laboratories around the world. Fractionation studies in mammalian cells indicated that the reduced pyridine nucleotide cofactor, NADH, is responsible for most MTT reduction. This was supported by studies using whole cells [9]. MTT reduction is therefore associated with metabolically active cells and is not only coupled with mitochondrial respiration, but also with the cytoplasm and non-mitochondrial membranes including the endosome/lysosome compartment and the plasma membrane [10]. The net positive charge on the MTT molecule is the primary factor for its cellular uptake via the plasma membrane potential.

Notably, the MTT assay may occasionally not accurately reflect the actual effect that a tested compound has on specific cells. However, the chemical structures or groups that may cause conflicting results in the MTT assay have not been identified. Recent studies have demonstrated that the MTT assay could underestimate of the anti-proliferative effect of (-)-epigallocatechin-3-gallate, the most abundant polyphenol in green tea [11]. In studies using the MTT assay to detect tumor chemosensitivity, anti-cancer chemotherapeutic agents, such as epirubicine, paclitaxel, doxetaxel, and imatinib mesylate (gleevec), showed increased cell viability [12,13]. Moreover, a number of studies have reported that certain plant extracts and redox-active polyphenols could interfere with the MTT assay as they directly reduce the MTT tetrazolium salt in the absence of cells [14].

The need to solubilize the MTT formazan crystals prior to spectrophotometric analysis in a microplate reader and the inherent endpoint nature of the reaction limit the use of the MTT assay to certain applications. This led to the development of tetrazolium analogues in which the phenyl moieties were decorated with negatively charged sulfonate groups, such as 2,3-bis-(2-methoxy-4-nitro5-sulfophenyl)-2H-tetrazolium-5-carboxanilide (XTT), a negatively charged inner salt [15] and 3-(4,5-dimethylthiazol-2-yl)-5-(3-carboxymethoxyphenyl)-2-(4-sulfophenyl)-2H-tetrazolium (MTS), a weakly acidic inner salt closely related to MTT [16]. These modifications resulted in the production of culture medium-soluble formazan products that eliminated the requirement of a solubilization step prior to quantitation. Correspondingly, as the increased negative charge on these molecules reduced their ability to move across cell membranes [17], intermediate electron acceptors (IEA), such as 1-methoxy-5-methyl phenazinium methylsulphate (1-methoxy PMS) were required to facilitate tetrazole dye reduction or to enhance the rate of reduction.

More recently, a new generation of water-soluble tetrazolium salts has been developed of which WST-1 is the prototype [18]. WST-1, a negatively charged disulfonated inner salt containing an iodine residue, is more stable than XTT and MTS in the presence of 1-methoxy PMS, its obligatory IEA. This led to the marketing of WST-1/1-methoxy PMS as a convenient single reagent cell proliferation kit. Several other tetrazolium salts in the WST series have been developed, the most useful of which perhaps being WST-8 [19]. WST-8 appears to have highly similar cellular reduction properties to WST-1 and is being marketed independently as the CCK- 8 assay. WST-8 is cell impermeable and is therefore reduced extracellularly, via electron transport from intracellular NADH to WST-8 across the plasma membrane mediated by 1-methoxy PMS. Although both MTT and WST-8/1-methoxy PMS reduction are driven by intracellular NADH, the source of NADH appears to differ within these two methods. WST-8/1-methoxy PMS reduction is more highly dependent on the malate/aspartate shuttle that links mitochondrial tricarboxylic acid cycle-NADH with the extramitochondrial space [20].

In preliminary experiments, we confirmed that compounds $\mathbf{1}, \mathbf{2}, \mathbf{3}$, and $\mathbf{4}$ could not directly reduce the MTT tetrazolium salt (not shown). In the present study, the direct influence of the compounds 
themselves with the reagent were eliminated by carefully washing the microplates with phosphate buffered saline (PBS) before MTT or CCK-8 solution was added, as indicated in the manufacturer's protocols. Nevertheless, the results obtained using the two methods were still contradictory (Figure 2). As expected, incubation of cells with compounds 1, 2, and $\mathbf{3}$ increased the reduction of the tetrazolium salt into purple formazan, compared with the control group (Figure 3), suggesting that mere reduction of the tetrazolium salt in the presence of a specific compound is not sufficient to judge the compound's ability to interfere with the MTT assay.

To determine whether compounds 1, 2, 3, and 4 could induce apoptosis in EA.hy926 cells, cellular morphological changes and phosphatidylserine externalization were assessed. Consistently with the decrease in cellular viability observed with the CCK-8 assay, the results of Hoechst staining and flow cytometry analysis using annexin V-FITC/PI suggested that the growth inhibition observed after compound 1, 2, and 3 treatment was due, at least in part, to EA.hy926 cell apoptosis. Our results also supported the idea that the caffeyl group present in both compounds $\mathbf{1}$ and $\mathbf{2}$ was responsible for the contradictory results obtained with the MTT assay.

Similar to our findings, Rottlerin, a potent large conductance potassium channel opener, did not exhibit reactivity toward MTT in vitro. However, it strongly enhanced the formation of formazan crystals inside cells, a result that was in evident conflict with Rottlerin inhibition of NF- $\mathrm{BB}$ and cell proliferation observed by analysis of $\left[{ }^{3} \mathrm{H}\right]$-thymidine incorporation into DNA [21]. The mechanism by which Rottlerin enhanced MTT reduction was attributed to its mitochondrial uncoupling effect [22]. Rottlerin has a cinnamoyl acid substituent group. Compared with cinnamoyl acid, compound $\mathbf{3}$ possesses two additional hydroxyl residues, which are located at C-3 and C-4. Therefore, we speculate that the mechanism by which compounds $\mathbf{1}$ and $\mathbf{2}$ cause conflicting results in the MTT assay may also be associated with their mitochondrial uncoupling effects. To test this hypothesis, comparative studies among compounds $\mathbf{1}, \mathbf{2}$, and a chemical uncoupler, such as trifluorocarbonylcyanide phenylhydrazone (FCCP), are needed.

\section{Experimental Section}

\subsection{Reagents and Chemicals}

Compound 3 (caffeic acid-powder, purity = 98.6\%) was purchased from Chengdu Push Bio-Technology Co., Ltd. (Chengdu, China). Compound 4 (3,4-dihydroxylphenylethanol-oil, purity $=98.5 \%$ ) was obtained from Chengdu Must Bio-Technology Co., Ltd. (Chengdu, China). MTT and dimethyl sulfoxide (DMSO) were obtained from Sigma-Aldrich (St. Louis, MO, USA). The CCK-8 assay was purchased from Dojin Laboratories (Kumamoto, Japan). Hoechst 33342 and annexin V-FITC/PI apoptosis detection kits were purchased from the Beyotime Institute of Biotechnology (Haimen, China).

\subsection{Plant Material}

C. tubulosa stems were collected from Yutian Prefecture, Xinjiang Uygur Autonomous Region, China. Voucher specimens were deposited in the Key Laboratory of High Altitude Medicine, Third 
Military Medical University (Chongqing, China) and authenticated by Yi Zhang from Chengdu University of Traditional Chinese Medicine (Chengdu, China).

\subsection{Extraction and Isolation of Plant Material}

Air-dried C. tubulosa $(0.6 \mathrm{~kg})$ stems were powdered and extracted with $50 \%$ ethanol for $2 \mathrm{~h}$ at $70{ }^{\circ} \mathrm{C}$. After removal of the solvent under reduced pressure, the ethanolic extract (212.5 g) was dissolved and suspended in water $(2 \mathrm{~L})$ and extracted with $n$-butanol $(2 \mathrm{~L} \times 3)$. The $n$-butanolic extract $(110 \mathrm{~g})$ was chromatographed on a polyamide column and eluted with ethanol/water $(0: 100,5: 95$, $15: 85,30: 70,50: 50)$ producing five fractions (A-E). Fraction B (65 g) was fractionated on an ODS column; elution with ethanol:water (1:9) separated compound 1 (3 g). Fraction D (10.5 g) was fractionated on an ODS column; elution with ethanol/water (1:4) separated compound 2 ( $1 \mathrm{~g})$.

NMR spectra were recorded on a Bruker Avance 600 spectrometer in $\mathrm{CD}_{3} \mathrm{OD}$ with Tetramethylsilane (TMS) as internal standard. Mass spectra were carried out on a BioTOF-Q mass spectrometer.

\subsection{Cell Culture}

EA.hy926 cell lines were obtained from the American Type Culture Collection (Manassas, VA, USA). Cells were cultured in RPMI 1640 medium, supplemented with $10 \%(\mathrm{v} / \mathrm{v})$ fetal bovine serum and $1 \%(\mathrm{v} / \mathrm{v})$ penicillin-streptomycin in a humidified environment with $5 \% \mathrm{CO}_{2}$ at $37{ }^{\circ} \mathrm{C}$.

\subsection{Cell Viability}

Cell viability was assessed using the MTT and CCK-8 assays. Compounds $\mathbf{1}$ and 2, and their aglycones, caffeic acid (3) and 3,4-dihydroxylphenylethanol (4) were dissolved in DMSO to the final DMSO concentration $<0.1 \%(\mathrm{v} / \mathrm{v})$ in culture media.

EA.hy926 cells were seeded on 96-well plates at $4 \times 10^{3}$ cells/well. After incubation for $24 \mathrm{~h}$, cells were treated with $6.25-50 \mu \mathrm{M}$ compound $1,2,3$, or 4 for $24 \mathrm{~h}$. Culture medium was removed and cells were washed twice with phosphate-buffered saline (PBS). Aliquots $(10 \mu \mathrm{L})$ of stock MTT solution $\left(5 \mathrm{mg} \cdot \mathrm{mL}^{-1}\right)$ were added to each well containing $100 \mu \mathrm{L}$ of medium and incubated with cells for $4 \mathrm{~h}$. After incubation, the medium was removed and $150 \mu \mathrm{L}$ aliquots of DMSO were added to each well to solubilize the formazan crystals. Absorbance was measured at $490 \mathrm{~nm}$ using a microplate reader (Bio-Tek Synergy HT, Winooski, VT, USA). Cell viability was expressed as the percentage of MTT reduction, assigning the $100 \%$ value to the absorbance of the control cells. All experiments were performed three times and presented as mean \pm standard deviation (SD).

The CCK-8 assay was performed following the literature with slight modifications [23]. Specifically, cells were treated with $6.25-50 \mu \mathrm{M}$ compounds $\mathbf{1}, \mathbf{2}, \mathbf{3}$, or $\mathbf{4}$ for $24 \mathrm{~h}$ and washed twice with PBS before the addition of $10 \mu \mathrm{L}$ CCK-8 solution and $100 \mu \mathrm{L}$ of culture media. After incubating the microplate at $37{ }^{\circ} \mathrm{C}$ for $2 \mathrm{~h}$, absorbance was measured at $450 \mathrm{~nm}$ using a microplate reader. Cell viability was expressed as the percentage of viable cells relative to control cells (100\%). All experiments were undertaken three times and expressed as mean $\pm \mathrm{SD}$. 


\subsection{Apoptosis Assessment by Hoechst 33342 Staining}

Apoptotic morphological changes were observed by Hoechst 33342 staining. Briefly, EA.hy926 cells were seeded on 48 -well plates $\left(2 \times 10^{4}\right.$ cells/well $)$ and treated with $50 \mu \mathrm{M}$ compound $\mathbf{1}, \mathbf{2}, \mathbf{3}$, or 4 for $48 \mathrm{~h}$ at $37{ }^{\circ} \mathrm{C}$, washed twice with PBS, and fixed with $4 \%(\mathrm{v} / \mathrm{v})$ paraformaldehyde for $15 \mathrm{~min}$ at room temperature. After rinsing twice with PBS, cells were stained with Hoechst $33342\left(10 \mu \mathrm{g} \cdot \mathrm{mL}^{-1}\right)$ for $5 \mathrm{~min}$ at room temperature, and washed twice with PBS. Hoechst-stained nuclei were visualized using a fluorescence microscope (Olympus, Tokyo, Japan).

\subsection{Apoptosis Analysis by Flow Cytometry}

Apoptotic cells were detected by annexin V-FITC/PI double-staining and flow cytometry analysis. Briefly, after treatment with $50 \mu \mathrm{M}$ compound 1, 2, 3, or 4 for $48 \mathrm{~h}$, cells were harvested and resuspended in PBS at $1 \times 10^{5}$ cells $\cdot \mathrm{mL}^{-1}$. After centrifugation at $500 \times g$ for $5 \mathrm{~min}$ at $25^{\circ} \mathrm{C}, 195 \mu \mathrm{L}$ of annexin V-FITC binding buffer and $5 \mu \mathrm{L}$ of annexin V-FITC were added. After gentle vortex-mixing, the mixture was incubated for $10 \mathrm{~min}$ at room temperature in the dark. After centrifugation at $500 \times \mathrm{g}$ for $5 \mathrm{~min}$ at $25^{\circ} \mathrm{C}, 190 \mu \mathrm{L}$ of FITC-conjugated annexin V binding buffer and $10 \mu \mathrm{L}$ PI were added. After gentle vortex-mixing, samples were analyzed using a FACSCalibur Flow Cytometer (Becton Dickinson, San Jose, CA, USA) within 30 min.

\subsection{Statistical Analysis}

All experimental data were expressed as mean \pm SD. The significance of the differences between experimental samples and the corresponding control was assessed by one-way analysis of variance (ANOVA) using the SPSS software (version 11.5). Statistical significance was set at $p<0.05$.

\section{Conclusions}

Our results suggest that overestimation of the number of viable cells observed the MTT assay could mask the cytotoxicity of certain compounds. Therefore, during drug screening processes, we recommend using a variety of methods to determine the effect of the specific compound in cell viability (such as CCK-8 and ${ }^{3} \mathrm{H}-\mathrm{TdR}$ assays) to avoid generating misleading results.

\section{Acknowledgments}

This work was supported by the China Postdoctoral Science Foundation (20100471774), the Research Fund of Education Bureau of Sichuan Province, China (13ZB0304), and the Science Development Fund of Chengdu University of Traditional Chinese Medicine (ZRMS201246).

\section{Author Contributions}

Yu-Jie Wang and Yu-Qi Gao designed the study. Yu-Jie Wang, Si-Min Zhou and Gang Xu conducted the study and wrote the manuscript. 


\section{Conflicts of Interest}

The authors declare no conflict of interest.

\section{References}

1. Kobayashi, H.; Oguchi, H.; Takizawa, N.; Miyase, T.; Ueno, A.; Usmanghani, K.; Ahmad, M. New phenylethanoid glycosides from Cistanche tubulosa (SCHRENK) HOOK. f. I. Chem. Pharm. Bull. 1987, 35, 3309-3314.

2. Editorial Committee of Chinese Pharmacopoeia. Chinese Pharmacopoeia; Medical Science and Technology Press: Beijing, China, 2010; Volume 1, p. 126.

3. Wuliya, Y.; Wang, X.W.; Zaoranmu, N. The protective effect of the glycosides of Cistanche on the cerebral hypoxia in mice. J. Xinjiang. Med. Univ. 2003, 26, 561-562.

4. Morikawa, T.; Pan, Y.N.; Ninomiya, K.; Imura, K.; Matsuda, H.; Yoshikawa, M.; Yuan, D.; Muraoka, O. Acylated phenylethanoid oligoglycosides with hepatoprotective activity from the desert plant Cistanche tubulosa. Bioorg. Med. Chem. 2010, 18, 1882-1890.

5. Li, L.; Tsao, R.; Yang, R.; Liu, C.M.; Young, J.C.; Zhu, H.H. Isolation and purification of phenylethanoid glycosides from Cistanche deserticola by high-speed counter-current chromatography. Food Chem. 2010, 108, 702-710.

6. Gong, Y.; Wu, J.; Huang, Y.F.; Shen, S.N.; Han, X.D. Nonylphenol induces apoptosis in rat testicular Sertoli cells via endoplasmic reticulum stress. Toxicol. Lett. 2009, 186, 84-95.

7. Jaruga, E.; Salvioli, S.; Dobrucki, J.; Chrul, S.; Bandorowicz-Pikula, J.; Sikora, E.; Franceschi, C.; Cossarizza, A.; Bartosz, G. Apoptosis-like, reversible changes in plasma membrane asymmetry and permeability, and transient modifications in mitochondrial membrane potential induced by curcumin in rat thymocytes. FEBS Lett. 1998, 433, 287-293.

8. Mosmann, T. Rapid colorimetric assay for cellular growth and survival: Application to proliferation and cytotoxicity assays. J. Immunol. Meth. 1983, 65, 55-63.

9. Berridge, M.V.; Herst, P.M.; Tan, A.S. Tetrazolium dyes as tools in cell biology: New insights into their cellular reduction. Biotechnol. Annu. Rev. 2005, 11, 127-152.

10. Liu, Y.B.; Peterson, D.A.; Kimura, H.; Schubert, D. Mechanism of cellular 3-(4,5dimethylthiazol-2-yl)-2,5-diphenyltetrazolium bromide (MTT) reduction. J. Neurochem. 1997, 69, 581-593.

11. Wang, P.W.; Henning, S.M.; Heber, D. Limitations of MTT and MTS-based assays for measurement of antiproliferative activity of green tea polyphenols. PLOS ONE 2010, 5, e10202.

12. Ulukaya, E.; Colakogullari, M.; Wood, E.J. Interference by anti-cancer chemotherapeutic agents in the MTT-tumor chemosensitivity assay. Chemotherapy 2004, 50, 43-50.

13. Sims, J.T.; Plattner, R. MTT assays cannot be utilized to study the effects of STI571/Gleevec on the viability of solid tumor cell lines. Cancer Chemother Pharmacol. 2009, 64, 629-633.

14. Bruggisser, R.; Daeniken, K.V.; Jundt, G.; Schaffner, W.; Tullberg-Reinert, H. Interference of plant extracts, phytoestrogens and antioxidants with the MTT tetrazolium assay. Planta Med. 2002, 68, 445-448. 
15. Paull, K.D.; Shoemaker, R.H.; Boyd, M.R.; Parsons, J.L.; Risbood, P.A.; Barbera, W.A.; Sharma, M.N.; Baker, D.C.; Hand, E.; Scudiero, D.A.; et al. The synthesis of XTT: A new tetrazolium reagent that is bioreducible to a water-soluble formazan. J. Heter. Chem. 1988, 25, 911-914.

16. Barltrop, J.A.; Owen, T.C; Cory, A.H.; Cory, J.G. 5-(3-Carboxymethoxyphenyl)-2-(4,5Dimethylthiazolyl)-3-(4-sulfophenyl)tetrazolium, inner salt (MTS) and related analogs of 3-(4,5-dimethylthiazolyl)-2,5-diphenyltetrazolium bromide (MTT) reducing to purple water-soluble formazans as cell-viability indicators. Bioorg. Med. Chem. Lett. 1991, 1, 611-614.

17. Marshall, N.A.; Goodwin, C.J.; Holt, S.J. A critical assessment of the use of microculture tetrazolium assays to measure cell growth and function. Growth Regul. 1995, 5, 69-84.

18. Ishiyama, M.; Shiga, M.; Sasamoto, K.; Mizoguchi, M.; He, P.G. A new sulfonated tetrazolium salt that produces a highly water-soluble formazan dye. Chem. Pharm. Bull. 1993, 41, 1118-1122.

19. Tominaga, H.; Ishiyama, M.; Ohseto, F.; Sasamoto, K.; Hamamoto, T.; Suzuki, K.; Watanabe, M. A water-soluble tetrazolium salt useful for colorimetric cell viability. Anal. Commun. 1999, 36, 47-50.

20. Tan, A.S.; Berridge, M.V. Tetrazolium dye reduction discriminates between mitochondrial and glycolytic metabolism. Redox Rep. 2004, 9, 302-307.

21. Torricelli, C.; Fortino, V.; Capurro, E.; Valacchi, G.; Pacini, A.; Muscettola, M.; Soucek, K.; Maioli, E. Rottlerin inhibits the nuclear factor kappaB/cyclin-D1 cascade in MCF-7 breast cancer cells. Life Sci. 2008, 82, 638-643.

22. Maioli, E.; Torricelli, C.; Fortino, V.; Carlucci, F.; Tommassini, V.; Pacini, A. Critical appraisal of the MTT assay in the presence of rottlerin and uncouplers. Biol. Proced. Online 2009, 11, 227-240.

23. Heng, B.C.; Zhao, X.X.; Xiong, S.J.; Ng, K.W.; Boey, F.Y.; Loo, J.S. Toxicity of zinc oxide $(\mathrm{ZnO})$ nanoparticles on human bronchial epithelial cells (BEAS-2B) is accentuated by oxidative stress. Food Chem. Toxicol. 2010, 48, 1762-1766.

Sample Availability: Samples of the compounds $\mathbf{1}$ and $\mathbf{2}$ are available from the authors.

(C) 2015 by the authors; licensee MDPI, Basel, Switzerland. This article is an open access article distributed under the terms and conditions of the Creative Commons Attribution license (http://creativecommons.org/licenses/by/4.0/). 